\title{
Virtud y reconocimiento: G.W.F. Hegel sobre la amistad*
}

\author{
Virtue and recognition: \\ G.W.F. Hegel on friendship
}

\author{
SERGIO CLAVERO GARCÍA \\ Instituto Cultura y Sociedad. Universidad de Navarra (Pamplona)
}

Recibido:14/06/2017_Aceptado: 16:06/2017

\begin{abstract}
RESUMEN
Al contrario que Aristóteles, Hegel dedica a la amistad tan solo unos pocos pasajes a lo largo de toda su obra. En dichos fragmentos la amistad aparece descrita como un tipo de relación basada exclusivamente en el sentimiento y el arbitrio individuales; como consecuencia, queda excluida de la esfera ética por carecer de una estructura institucional y depender únicamente de la opinión particular. A pesar de todo, Hegel admite que la amistad representa una realización genuina y valiosa de la libertad, en la medida en que en ella se produce un reconocimiento mutuo que permite a cada sujeto estar-consigo-mismo-en-el-otro.

PALABRAS CLAVE

AMISTAD, HEGEL, LIBERTAD, RECONOCIMIENTO, SENTIMIENTO, VIRTUD.

\section{ABSTRACT}

In contrast to Aristotle, Hegel devotes to friendship only a few passages along his works. In those fragments, friendship is portrayed as a type of relationship based exclusively on the feeling and the will of the individual. As a consequence, it is excluded from the ethical sphere, since it lacks an institutional structure and depends wholly upon particular opinion. Nevertheless,

* Agradezco especialmente a Miguel Martí sus muchas contribuciones durante las largas horas de animada discusión sobre el tema Mi profundo agradecimiento también a los profesores Juan José Padial, Javier Hernández Pacheco, Jesús de Garay, Cristóbal Holzapfel y Manuel Barrios: aunque me ha sido imposible incluir aquí todas sus valiosas observaciones, sus comentarios me han ayudado a profundizar en el tema y me han abierto caminos que espero poder explorar en el futuro.
\end{abstract}

(C) Contrastes. Revista Internacional de Filosofia, vol. XXII-N² (2017), pp. 165-174. ISSN: 1136-4076

Departamento de Filosofía, Universidad de Málaga, Facultad de Filosofía y Letras Campus de Teatinos, E-29071 Málaga (España) 
Hegel acknowledges that friendship represents a genuine and valuable realization of freedom, insofar as it implies a mutual recognition that allows each subject to be-with-herself-in-another. KEYWORDS

FEELING, FREEDOM, FRIENDSHIP, HEGEL, RECOGNITION, VIRTUE.

\section{INTRODUCCIÓN}

Christiane Luise Hegel recordaba a su hermano mayor como un joven torpe en el arte de bailar, pero rodeado de muchos amigos (Pinkard 2001, pp. 40-1). Él mismo empleó esa palabra («amigo») para referirse a diversos conocidos suyos, tanto en sus cartas (cf. Hegel 1952; 1953; 1969) como en los libros que publicó ( $c f$. $\mathrm{PFD}^{1} \S 203, \mathrm{Obs).} \mathrm{Sin} \mathrm{embargo,} \mathrm{su} \mathrm{obra} \mathrm{carece} \mathrm{por} \mathrm{completo}$ de un estudio de la amistad como tal. La ausencia de dicho análisis sorprende aún más si tenemos en cuenta la declarada admiración de Hegel por Aristóteles (Enc. ${ }^{2} \S 378 ;$ PFD $\S 140$, nota), quien sí reserva a la amistad un lugar preeminente dentro de su doctrina ética.

Las referencias a la amistad son muy escasas en los textos hegelianos. Esta parvedad ha llevado a interpretaciones contradictorias sobre cómo concebir la amistad desde la óptica de Hegel y qué lugar ocuparía esta (si es que puede ocupar alguno) dentro del sistema. Así, por ejemplo, para Adriaan Peperzak, «la amistad, en el sentido aristotélico de philia, no juega ningún papel en la filosofía práctica de Hegel» (Peperzak 2001, pp. 648-9); Robert R. Williams, en cambio, considera que la estructura del reconocimiento recíproco en Hegel es similar a la intersubjetividad implícita en la teoría aristotélica de la amistad (Williams 2012, pp. 55 y ss).

Cada una de las diversas exégesis basa sus conclusiones, bien en la casi total ausencia de textos hegelianos dedicados a la amistad, bien en el análisis de alguno de los escasos fragmentos que mencionan el tema; cuál sea ese fragmento escogido y supuestamente clave para la interpretación varía según a qué comentarista atendamos.

El objetivo del presente escrito es precisamente realizar un breve análisis de alguno de dichos pasajes (me centraré concretamente en dos), con un doble fin:

1) en primer lugar, entender qué características se le pueden atribuir a las relaciones de amistad desde la perspectiva hegeliana;

1 Principios de la Filosofía del Derecho (en lo sucesivo PFD), 1999 (ver bibliografía final).

2 Enciclopedia de las ciencias filosóficas (en los sucesivo Enc.), 2017 (ver bibliografía final). 
2) y en segundo lugar, intentar explicar el aparente olvido del tema por parte del propio Hegel.

\section{LA AMISTAD COMO RELACIÓN DE RECONOCIMIENTO Y FUENTE DE LIBERTAD}

La primera alusión relevante a la cuestión de la amistad que me gustaría mencionar pertenece al agregado del parágrafo 7 de los Principios de la Filosofía del Derecho (una alusión, por tanto, que debe tomarse con todas las precauciones correspondientes a un texto que no es de puño y letra del propio Hegel, sino un añadido posterior). El texto dice así:

«Esta libertad [la del tercer momento de la voluntad, en el que se unen universalidad y particularidad] la tenemos ya en la forma del sentimiento, por ejemplo, en la amistad y en el amor. En estos casos el hombre no está unilateralmente dentro de sí, sino que sólo al considerar al otro como otro se tiene el sentimiento de sí mismo» (PFD, §7, Agr).

Del citado pasaje pueden extraerse tres ideas relevantes sobre la naturaleza de las relaciones de amistad:

1) En primer lugar, es una forma de libertad, de estar-conmigo-mismoen-el-otro. De hecho, Axel Honneth se apoya en este pasaje para afirmar que "[Hegel] está convencido de que sólo las relaciones comunicativas que se efectúan según el modelo de la amistad permiten efectivamente la realización de su libertad al sujeto individual» (Honneth 2016, pp. 77-8). Conviene resaltar un matiz importante: Honneth habla de las relaciones que siguen «el modelo de la amistad». Es decir, aunque en la amistad el individuo realiza su libertad, hay otras formas de relación en las que también lo hace. En otras palabras, el sujeto es libre no sólo en la relación con sus amigos, sino en todo vínculo que siga el modelo de las relaciones de amistad.

2) La segunda idea relevante que podemos extraer del fragmento de Hegel es precisamente qué tipo de relación se da en la amistad: esa libertad, ese no estar unilateralmente en mí sino estar-conmigo-mismo-en-el-otro, presenta una estructura de reconocimiento mutuo. Como se afirmaba en el texto, «sólo al considerar al otro como otro se tiene el sentimiento de sí mismo»; es decir, sólo al reconocer al otro como tal alcanzo a reconocerme a mí mismo. Esta consideración es la que según Williams acerca las posturas de Hegel y Aristóteles (Williams 2012, p. 65), junto a la necesidad de que el reconocimiento sea recíproco y consciente ( $\mathrm{EN}^{3}$ VIII 2, 1155b27-1156a5).

3) En tercer lugar, este primer fragmento de Hegel deja traslucir claramente cómo la libertad realizada en la amistad se experimenta en la forma del sentimiento. Sobre esto volveré más adelante, pues es determinante a la hora de entender por qué la amistad no recibe un tratamiento específico dentro del

3 Ética a Nicómaco (en lo sucesivo EN), 2014 (ver bibliografía final). 
apartado dedicado a la ética de los Principios de la Filosofía del derecho, como sí ocurre, por ejemplo, con la familia de estilo burgués.

\section{LA CONTINGENCIA DE LA OPINIÓN Y EL SENTIMIENTO}

El segundo pasaje de Hegel dedicado a la amistad que me parece importante resaltar dice así:

«En el caso de la amistad, [...] depende enteramente de mí decidir en qué individuo deseo estar interesado. Esto concierne a la particularidad de mi carácter y mi modo de ser, los cuales son contingentes. Y la propia amistad es algo contingente [...] ¿Por qué no está la amistad entre las virtudes prescritas por Cristo? [...] Con los griegos encontramos unas pocas amistades ejemplares, las cuales destacan tanto precisamente porque son poco frecuentes» $\left[\mathrm{VSG}^{4}\right.$ p. 897]

Es decir, la amistad es para Hegel algo arbitrario, que no puede ser prescrito como obligatorio, sino que depende por completo de la opinión y el deseo de cada individuo. En este sentido, se trata de un tipo de relación contingente, cuya propia existencia está subordinada a mi carácter e incluso a las circunstancias, y que en su grado perfecto resulta por tanto poco frecuente (ya Aristóteles señalaba que es natural que las amistades perfectas sean raras [EN VIII 3, 1156b25]). Volveré sobre estas ideas más adelante.

Una vez comentados estos dos pasajes, podemos preguntarnos por qué Hegel no dedica más espacio a tratar un tema tan relevante como el de las relaciones entre amigos. La respuesta pasa, a mi entender, por una de las características principales de la amistad, que se deduce de lo expuesto hasta ahora: como señala Michael Hardimon, la amistad no es una institución en sentido propio, no es una estructura que se autorreproduzca o que exista más allá de los propios individuos que la forman; la existencia de una amistad depende, como ya se ha dicho, única y exclusivamente del arbitrio de las dos personas implicadas (Hardimon 1994, p. 336).

Podría replicarse que lo mismo ocurre con el amor entre marido y mujer, al que Hegel sí que dedica muchas páginas, pero en realidad existe una diferencia fundamental: como señala el propio Hegel, en el caso del matrimonio hay una declaración solemne del consentimiento mutuo que permite el reconocimiento por parte de la comunidad, y la unión matrimonial sólo es ética si se produce dicha ceremonia (PFD, §164). En otras palabras, el matrimonio

4 Vorlesungen über die Philosophie des subjektiven Geistes (en lo sucesivo VSG), 2011 (ver bibliografía final). No existe hasta ahora traducción al español de esta obra de Hegel (próximamente aparecerá una a cargo de Juan José Padial), por lo que la aquí presentada es obra mía; para realizarla me he apoyado en la excelente versión inglesa publicada en 2007 (ver bibliografía final) por Robert R. Williams a partir de la edición alemana de Franz Hespe y Burkhard Tuschling. 
alcanza su auténtico estatuto ético cuando la comunidad lo reconoce como tal. Esto no ocurre ni puede ocurrir en el caso de la amistad, puesto que no existe ninguna ceremonia (al menos en el caso de las amistades modernas) por la que dos personas declaren solemnemente su amistad a los ojos de toda la sociedad. La necesidad del reconocimiento social (y no meramente el de los individuos involucrados) para que una relación sea verdaderamente ética es una característica fundamental del espíritu objetivo en la perspectiva hegeliana. En este sentido, Hegel considera que el mundo ético no puede ser entregado a la contingencia de la opinión y el arbitrio subjetivos; el sentimiento no puede arrogarse el papel que corresponde a la razón (PFD pp. 52-3).

\section{LA AMISTAD, FUERA DE LA ESFERA ÉTICA}

La amistad, por tanto, se inscribe en este ámbito del sentimiento y el arbitrio subjetivos. De este hecho se desprenden tres consecuencias relevantes (y fuertemente interrelacionadas entre sí) para nuestra discusión:

1) En primer lugar, la amistad no pertenece al ámbito de la ética, sino al de la moralidad. En otras palabras, se trata de una cuestión individual, librada a la libertad de la conciencia moral de cada uno. Tener amigos es una forma de realizar la propia libertad, como ya he mencionado, y por tanto en un Estado racional se darán las condiciones necesarias para que este tipo de relaciones puedan ocurrir. Ahora bien, cuántos sean esos amigos, qué obligaciones lleve consigo esa relación y cuándo pueda darse esta por concluida son factores que dependen exclusivamente de los individuos implicados.

Esto no quiere decir, como interpreta Pedro Laín Entralgo, «que [para Hegel] en una sociedad donde tenga plena realidad el 'reino de la razón' [...] la amistad tradicional sólo podría ser una de estas dos cosas: un sinsentido o un delito, un sentimiento nostálgico o una infracción de la ley» (Laín Entralgo 1986, p. 121). Difícilmente la realización de la propia libertad es un sinsentido, y la amistad constituirá una invitación al delito sólo cuando pueda interferir en el correcto funcionamiento de las instituciones éticas (por ejemplo, si un juez se siente inclinado a favorecer injustamente a alguien por el hecho de ser su amigo). En definitiva, la amistad está permitida en el Estado racional, como realización del bien a los ojos de la conciencia individual. Es decir, tener amigos forma parte de la libertad de la conciencia moral de cada uno: quien quiera puede tenerlos, pero no se puede exigir a nadie que los tenga o que sea buen amigo.

Como veíamos más arriba, la amistad es algo contingente y completamente arbitrario, de modo que a lo ético no le compete prescribir quién puede o debe ser amigo de quién ni cuándo dos personas son o no amigas; tampoco puede determinar cuáles son los deberes implicados en esa relación, como sí ocurre en la sociedad civil, en la que la corporación tiene exigencias legítimas sobre 
sus miembros (PFD $\$ 238$ y $§ 253$ ). La amistad queda así fuera de la esfera de influencia de los poderes éticos, y por tanto, de lo ético como sistema de las determinaciones de la idea (ibid. \$145).

2) En segundo lugar, como ya se ha mencionado, cuándo empieza y cuándo acaba una amistad depende enteramente del arbitrio de los implicados. Siendo además una relación en la que el sentimiento juega un papel tan importante, y dado que no cabe una institucionalización que garantice o al menos favorezca su durabilidad, cualquier amistad es por definición inestable.

Ya Aristóteles señalaba que las amistades basadas en el placer y en la utilidad son de suyo muy mutables y acaban por no ser duraderas en muchos casos (EN VIII 3, 1156a15 y ss). En un parágrafo de los escritos de la época de Nüremberg, Hegel parece sugerir precisamente una lectura utilitarista de la amistad: «La amistad se basa en la igualdad de caracteres, en especial el interés en realizar una obra en común con otro, y no en el deleite en la persona del otro como tal» (Hegel 2006, §67). Esta afirmación parece apoyar la interpretación de Laín Entralgo, para quien Hegel reduce la amistad a mera camaradería (Laín Entralgo 1986, p. 121).

Sin embargo, las frases con las que continúa la cita anterior arrojan alguna duda sobre el carácter meramente utilitario de la amistad: «Se debe resultar lo menos molesto posible al amigo. Lo más delicado es no pedirles que nos presten ningún servicio. Uno no debe ahorrarse a sí mismo las cosas para cargarlas sobre los demás» (Hegel 2006, §67). Si no se debe molestar a los amigos salvo que sea inevitable, difícilmente se los puede considerar un mero medio para alcanzar la satisfacción de un interés común: dicho interés o actividad no es lo más importante, pues parece que primero hay que tomar en consideración hasta qué punto supone una molestia para el amigo en una circunstancia concreta.

Dejando a un lado las amistades por placer y por utilidad, que como hemos indicado son muy inestables, para Aristóteles las amistades basadas en la virtud sí son más permanentes, puesto que la propia virtud es una disposición difícil de relegar (cf. EN VIII 6, 1158b10; VIII 8, 1159b1-10). En mi opinión aquí se encuentra el núcleo de la diferencia entre el planteamiento aristotélico y el hegeliano: mientras que para Aristóteles la virtud es una disposición personal del carácter ( $c f$. EN II 5, 1106a10 y ss), para Hegel la virtud es «lo ético, en tanto se refleja en el carácter individual determinado por la naturaleza» (PFD §150).

En otras palabras, desde la perspectiva hegeliana la virtud no puede darse desligada de lo ético. De lo contrario, su contenido sería determinado meramente por el individuo, con el riesgo inevitable de caer en el subjetivismo y el relativismo moral; su su misma permanencia sería una cuestión de facto, siempre amenazando con disolverse en función del arbitrio y la opinión individual. El propio Hegel afirma lo siguiente a propósito de las relaciones de amistad, en la continuación de uno de los textos citados al inicio: 
«La compasión, el agradecimiento, etc. se refieren a aquellas relaciones que no pueden ser constituidas por las determinaciones éticas, aunque se relacionan de forma general con lo ético; de modo que incluyen un interés en el bienestar del otro, pero de manera que el tipo y la extensión de ese interés permanecen más bien como algo dependiente de las circunstancias y de las ocasiones especiales. En todos estos asuntos, la particularidad entra en juego en mayor o menor medida, de acuerdo con lo cual las cosas son dejadas a la contingencia, la particularidad y la arbitrariedad» $\left(\mathrm{VSG}^{5}\right.$ p. 898).

Dicho de otro modo, la amistad depende del deseo del individuo y de la contingencia de las circunstancias. Recordemos en este sentido cómo el propio Aristóteles también advierte algunos factores circunstanciales que pueden afectar a la amistad: por ejemplo, la amistad requiere de tiempo para poder llegar a una cierta intimidad (EN VIII 6, 1158a10-5), y si se da una ausencia muy prologada, puede caer en el olvido (EN VIII 5, $1157 \mathrm{~b} 10$ y ss). Esta influencia de las circunstancias contingentes provoca la desconfianza de Hegel, junto al hecho de que el peso de la amistad recaiga exclusivamente sobre el arbitrio individual. Su solución pasa por privilegiar en su teoría aquellas instituciones que están jurídicamente constituidas, como señala Honneth (2016, pp. 142 y 144).

Hegel pone el énfasis en dichas instituciones porque considera que son las que permiten una auténtica transición entre ética y política. Es cierto que Aristóteles también defiende la relación estrecha entre ética y política (EN, X 9, 1179b1 y ss), pero Hegel va más allá: ambas están intrínsecamente unidas, de modo que solo es ético lo que tiene una dimensión político-social. Cualquier rasgo de carácter que sea (podríamos decir) meramente moral, por mucho que sea lícito y pueda contribuir a la felicidad de un individuo, no puede considerarse una virtud ética en sentido propio, pues no tiene una traducción político-social; no es institucionalizable, de modo que ni recibe su contenido de la sustancia ética ni ayuda a sostenerla.

3) Lo cual nos lleva a la tercera de las consecuencias derivadas de considerar la amistad como una disposición no institucionalizable. Esa consecuencia es la siguiente: como acabo de mencionar, la amistad no contribuye a la autorreproducción de la sustancia ética.

Pensemos por un momento en los tres estadios de dicha sustancia ética: en la familia, nuevos individuos (nuevos ciudadanos) vienen al mundo (PFD $\S 173)$; en la sociedad civil, esos individuos alcanzan sus medios de subsistencia y la satisfacción de sus necesidades en interdependencia mutua (ibid. \$189 y ss); en el Estado, que es condición de posibilidad de los dos estadios anteriores, los intereses particulares son retrotraídos de nuevo a la unidad sustancial (ibid.

5 Ver nota $n^{\circ} 5$. 
§259), de modo que los individuos se orienten al bien común y la subsistencia del todo social.

En cambio, la amistad, aunque representa una forma de realizar la libertad del individuo, no contribuye a la autosustentación del todo ético. Al no ser institucionalizable, su contenido, continuidad, disolución, etc. (incluso su propia existencia) queda completamente al albur del deseo particular de los individuos involucrados. Y ya hemos mencionado anteriormente que el mundo ético no puede depender únicamente del arbitrio particular y la opinión subjetiva.

Esta misma circunstancia explica, a mi entender, que en sus Principios de la Filosofía del Derecho Hegel se limite a considerar unos tipos concretos de vinculación, dejando fuera otros que también son aparentemente relevantes, como las relaciones entre hermanos (por poner solo un ejemplo): el contenido de dichas relaciones se enmarca más bien en la esfera de la moralidad, y aunque quepa dar indicaciones al respecto, e incluso se admita que pueden contribuir a la felicidad y libertad de los individuos, no forman parte de los momentos esenciales de la sustancia ética.

\section{Conclusión}

En resumen, para Hegel la amistad es un tipo de relación moralmente valiosa en la que el individuo puede alcanzar cierto reconocimiento y actualizar su libertad (es decir, estar-consigo-mismo-en-el-otro). Ahora bien, lo hace en la forma del sentimiento, en la esfera del arbitrio subjetivo, esto es, de modo no equiparable al de las instituciones éticas. ¿Cabe por tanto incluir la amistad dentro del sistema? ${ }^{6}$ Atendiendo a los textos de Hegel, la respuesta es sí, pero dentro de la esfera de la moral, no de la ética.

Para hacer esto último (considerarla como una relación ética) tiene que mostrarse que la amistad admite al menos un cierto grado de institucionalización, de reconocimiento e influencia por parte de la comunidad. Esta es por ejemplo la línea que sigue Axel Honneth cuando critica a Hegel por haberse centrado demasiado en la familia de estilo burgués y haber dejado de lado otros tipos de relaciones normativas de trazo sentimental como la amistad: para Honneth, somos capaces de distinguir entre amistades verdaderas y falsas con base en criterios o prácticas sociales, y consideramos que las «reglas» que rigen nuestras relaciones de amistad existen también fuera de esta, en la sociedad (Honneth 2014, p. 178); en este sentido, la amistad sí sería para Honneth un tipo de vinculación ética, reconocida y respaldada socialmente.

6 Más concretamente, la pregunta sería si la amistad puede incluirse dentro del ámbito del espíritu objetivo, dado que Hegel sí trata el tema (aunque sea brevemente) en sus Lecciones sobre el espíritu subjetivo, como puede apreciarse en los textos citados más arriba. 
Pero para Hegel, la amistad pertenece al ámbito del sentimiento y del arbitrio subjetivo, y ahí debe quedarse, sin influir en las relaciones éticas entre los individuos.

\section{REFERENCIAS BIBLIOGRÁFICAS}

ARISTÓTELES 2014: Ética a Nicómaco, edición bilingüe y traducción de María Araujo y Julián Marías. Madrid: Centro de Estudios Políticos y Constitucionales, $10^{\mathrm{a}} \mathrm{ed}$.

HARDIMON, MICHAEL O. 1994: «Role Obligations», The Journal of Philosophy, Vol. 91, No. 7 (Jul., 1994), pp. 333-363.

HEGEL, G.W.F. 1952: Briefe von und an Hegel. I, 1785-1812, herausgegeben von Johannes Hoffmeister. Hamburg: Meiner.

, 1953: Briefe von und an Hegel. II, 1813-1822, herausgegeben von Johannes Hoffmeister. Hamburg: Meiner.

, 1969: Briefe von und an Hegel. III, 1823-1831, herausgegeben von Johannes Hoffmeister. Hamburg: Meiner.

, 1999: Principios de la filosofía del derecho o Derecho natural y ciencia política, traducción y prólogo de Juan Luis Vermal. Barcelona: Edhasa, $2^{\mathrm{a}}$ ed.

-, 2006: «Unterklasse Rechts- Pflichten- und Religions-Lehre aus den Schuljahren 1809/10 bis 1815/16", en Gesammelte Werke, Band 10,1: Nürnberger Gymnasialkurse und Gymnasialreden (1808-1816), herausgegeben von Klaus Grotsch. Hamburg: Felix Meiner.

___ 2007: Lectures on the philosophy of spirit: 1827-8, translated and with and introduction by Robert R. Williams. Oxford: Oxford University Press.

-, 2011: «Wintersemester 1827/28. Nachschrift Stolzenberg mit Varianten aus den Nachschriften Johann Eduard Erdmann und Ferdinand Walter», en Gesammelte Werke, Band 25,2: Vorlesungen über die Philosophie des subjektiven Geistes, herausgegeben von Christoph Johannes Bauer. Hamburg: Felix Meiner.

___ 2017: Enciclopedia de las ciencias filosóficas [1830], edición bilingüe, introducción, traducción y notas de Ramón Valls Plana. Madrid: Abada.

HONNETH, A. 2014: El Derecho de la Libertad: esbozo de una eticidad democrática, traducido por Graciela Calderón. Madrid: Clave Intelectual.

, 2016: Sufriendo de indeterminación, en A. Honneth, Patologias de la libertad, traducción e introducción de Francesc J. Hernàndez y Benno Herzog. Buenos Aires: Las cuarenta.

LAIIN ENTRALGO, P. 1986: Sobre la amistad. Madrid: Espasa-Calpe, $2^{\mathrm{a}}$ ed.

PEPERZAK, ADRIAAN T. 2001: Modern freedom: Hegel's legal, moral, and political philosophy. Boston: Kluwer Academic Publishers. 
PINKARD, T. 2001: Hegel: una biografía, traducción de Carmen García-Trevijano Forte. Madrid: Acento.

WILLIAMS, ROBERT R. 2012: Tragedy, recognition, and the death of God: studies in Hegel and Nietzsche. Oxford: Oxford University Press.

Sergio Clavero García es investigador predoctoral en el Instituto Cultura y Sociedad de la Universidad de Navarra

Líneas de investigación:

Filosofía social hegeliana; teoría hegeliana del reconocimiento; conexión entre vínculos sociales y obligaciones morales.

Últimas publicaciones:

(Recensión) S. Hahn y B. Wiker: "Dawkins en observación. Una crítica al nuevo ateísmo", Anuario Filosófico XLIV/3 (2011), 632-635.

Correo electrónico: sclavero@alumni.unav.es 